\title{
Tratamiento de pacientes parcialmente edéntulos con prótesis dentoimplantosoportadas
}

\author{
CAÑADA MADINAZCOITIA L * \\ DEL RÍO HIGHSMITH J ** \\ MARTÍNEZ VÁZQUEZ DE PARGA J ***
}

Cañada Madinazcoitia L, Del Río Highsmith J, Martínez Vázquez de Parga J. Tratamiento de pacientes parcialmente edéntulos con prótesis dentoimplantosoportadas. Av Periodon Implantol. 2001; 13, 1: 9-17

\begin{abstract}
RESUMEN
Un problema con el que nos encontramos cuando hacemos la planificación del paciente parcialmente desdentado, es si al colocar los implantes sería correcto unirlos a los dientes adyacentes o no. Este dilema se debe a la diferente movilidad que tienen el diente natural con su ligamento periodontal y el implante osteointegrado. Brunski publicó estas diferencias en intrusión del diente y el implante.
\end{abstract}

Sin embargo, hay numerosos beneficios de unir ambos pilares, entre los que podemos citar: necesitaremos menor número de implantes, lo cual puede ser determinante para poder tratar al paciente o no, tendrá un coste económico menor; se obtendrá una mejor propiocepción de la restauración que si la prótesis es de soporte exclusivamente implantario.

En el presente artículo hacemos una amplia revisión bibliográfica que nos indique qué soporte científico hay para poder utilizar clínicamente la conexión diente con implante y, si se puede, cómo debe ser esta unión, rígida o no rígida. Tenemos que tener en cuenta que hay muchas variables a considerar en este tipo de pacientes en los que vamos a unir un diente con un implante, por eso deberemos ser cuidadosos a la hora de extrapolar los resultados publicados a todas las situaciones clínicas que se nos presentan en nuestras consultas, porque la distribución de cargas varía de una forma significativa en función del estado periodontal del diente y del grado de osteointegración del implante. Es decir, para la decisión final de qué tipo de tratamiento debo utilizar, será el clínico el que tendrá que sopesar la situación de los diferentes pilares antes de tomar la decisión final.

\section{PALABRAS CLAVE}

Implantes, prótesis parciales fijas, unión diente implante.

\section{INTRODUCCIÓN}

Desde que se comenzó a utilizar clínicamente los implantes, surgió la cuestión de la conveniencia o no de unir los implantes osteointegrados a los dientes naturales mediante una prótesis fija. Según Branemark (1), debemos separar el soporte implantario del soporte dentario, desde un punto de vista teórico se rechazó la posibilidad de unir diente con implante debido a la diferencia de comportamiento frente las cargas oclusales porque como señala Cohen (2)el diente dispone de un ligamento periodontal que le permite intruirse dentro del alveolo entre 50 y 200 micras mientras que el implante osteointegrado al estar rígidamente unido al hueso solo lo hace unas 10 micras, de esto se deducía que las cargas recaerían

\footnotetext{
* Profesor Asociado.

** Catedrático.

*** Profesor titular.
} 


\section{AVANCES}

Volumen 13 - $\mathrm{N}^{\circ} 1$ - Abril 2001

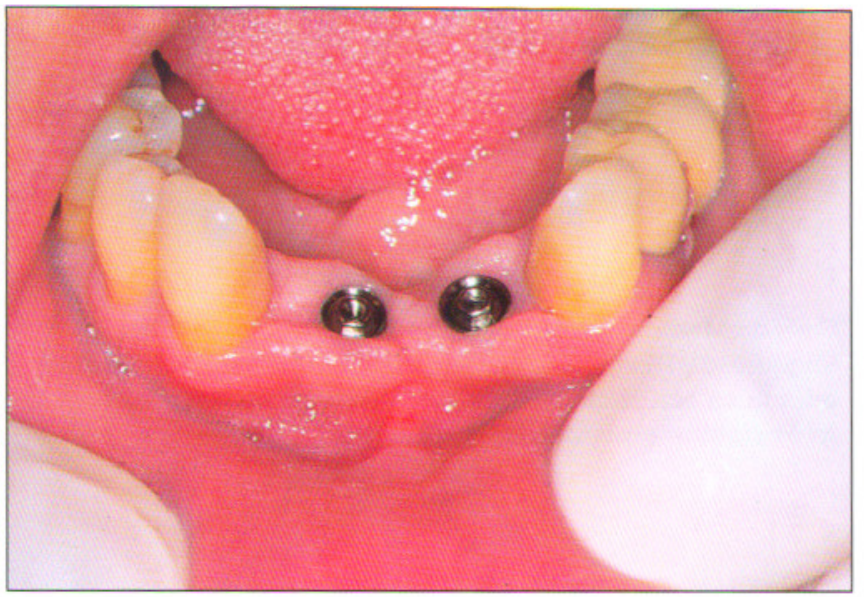

Fig. 1.: Dos implantes para soportar cuatro incisivos inferiores.

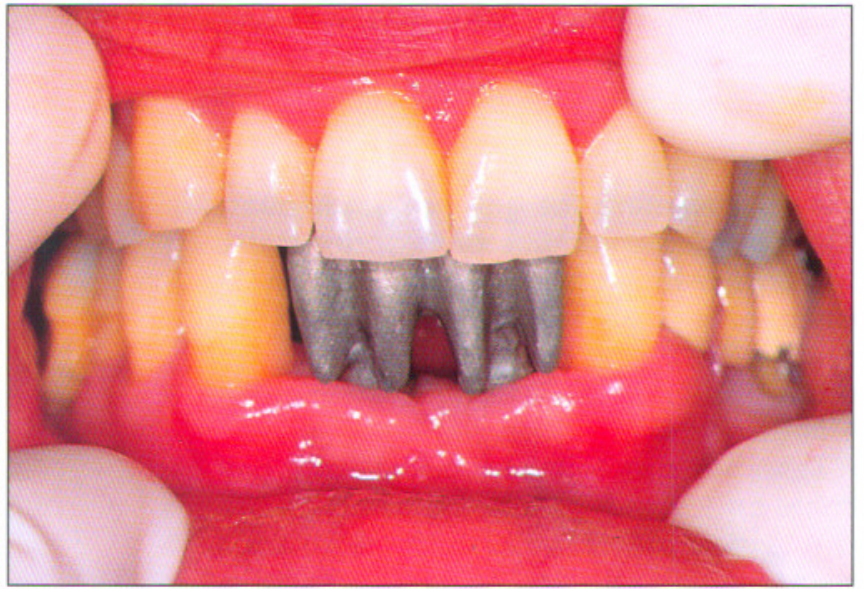

Fig. 2.: Soporte exclusivamente implantario.

fundamentalmente en el implante y esto ocasionaría fracturas y aflojamientos de los tornillos de las prótesis e incluso la perdida de la osteointegracion como señalan Schitman 1988 (3), Brunski 1988 (4) y Skalak 1985 (5). Según algunos autores como Uhlrich 1993 (6) y Hammerle 1995 (7) los pacientes con implantes, al carecer estos de ligamento periodontal y por lo tanto de receptores táctiles puede ocasionar que estos pacientes realicen una mayor fuerza masticatoria que antes de perder sus dientes. Hay numerosos estudios in vitro y estudios de análisis matemáticos que demostraban esta hipótesis, entre los que cabe destacar. ( Kirsch y Ackermann,1989 (8); Richter, 1989 (9); Richter, Orshall y Jovanovic,1990 (10); Valentin et al. , 1990 (11); van Rossen et al., 1990 (12). Tratando de compensar esta diferencia de resilencia se han desarrollado diferentes procedimientos, como por ejemplo, los implantes IMZ que se diseñaron con una pieza intramovil, que compensara de alguna manera esta diferencia (13). Sin embargo, en algunas ocasiones en clínica nos hemos visto "obligados" a unir diente con implante y observábamos que no se producía sobre-

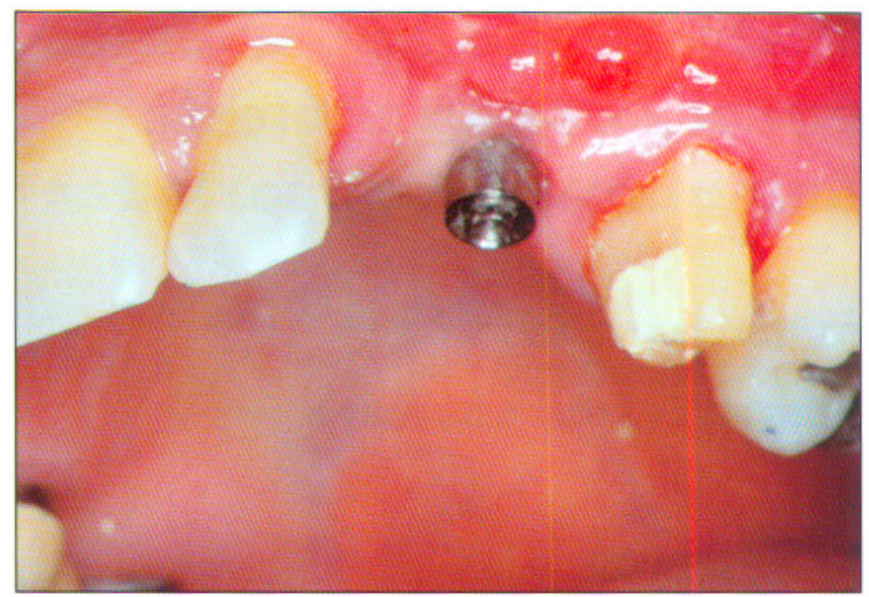

Fig. 3.: Un solo implante, ausencia de canino y primer premolar.

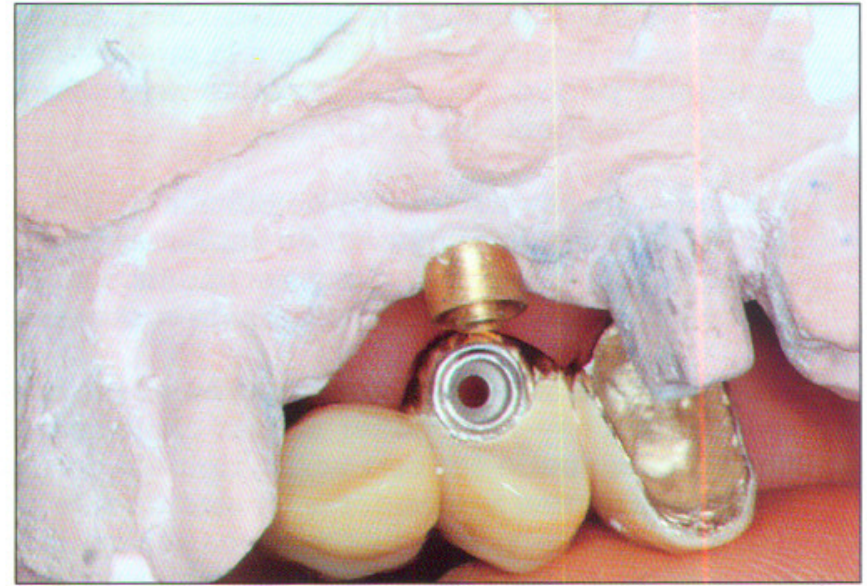

Fig. 4.: Soporte mixto dentoimplantario.

carga de consecuencias negativas sobre los dientes ni los implantes, y estos casos cumplían su función correctamente.

En estas situaciones en que teníamos que utilizar un soporte mixto de nuestras prótesis, se nos planteaba la cuestión de cómo unirlos, para ello disponíamos de varias alternativas.

\section{TIPOS DE UNIÓN DIENTE IMPLANTE}

\section{CONECTORES RÍGIDOS}

Se feruliza el diente y el implante, podemos hacerlo con una estructura metálica única, cementada en ambos pilares o cementada en el diente y atornillada en el implante (14). En otras ocasiones, algunos autores hacen dos estructuras metálicas independientes, 


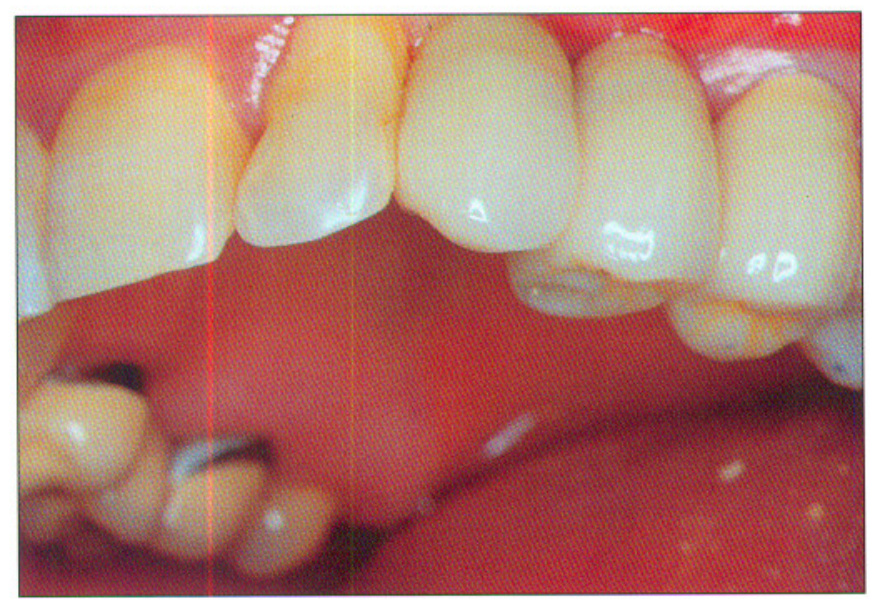

Fig. 5.: Caso terminado. Unión rígida diente e implante.

una ira atornillada en el implante y la otra cementada en el diente y a su vez ambas estructuras estarán atornilladas entre si, mediante un atache rígido (15). Este es un procedimiento mas complejo y caro que el hacer una sola estructura, y además puede introducir tensiones entre los pilares si el ajuste de este atache de precisión no es correcto, posiblemente su utilización ha sido mas debida a la desconfianza en la conexión rígida entre los dos pilares, que de esta forma si identificábamos algún problema en el diente o en el implante pudiéramos independizar el soporte. También podríamos utilizar esta conexión cuando tenemos dudas de la viabilidad a largo plazo del diente o del implante.Actualmente, y así lo proponía Lindh en 1997 (16), teniendo en cuenta que las complicaciones graves de los implantes son relativamente pequeñas, que el porcentaje de supervivencia de los implantes en los casos parciales es alta y que el coste económico es mucho menor, nos hace concluir que la mejor alternativa es hacer la estructura en una sola pieza. Si surge una complicación grave actuaremos como procederíamos en un caso similar en prótesis fija sobre dientes naturales, es decir, seccionaríamos la prótesis y una vez solucionado el problema haríamos una nueva prótesis.

En ocasiones proponemos realizar conexiones rigidas desmontables (atornilladas) por razones estrategicas, especialmente en situaciones donde el diente esta endodonciado y anquilosado al hueso, lo que en teoria favorece el comportamiento biomecánico aunque no esta demostrado clínicamente, por lo que la endodoncia intencionada es discutible en estos casos.

\section{COFIAS TELESCÓPICAS}

Esta alternativa de unión diente implante consiste en realizar una cofia metálica de recubrimiento total al

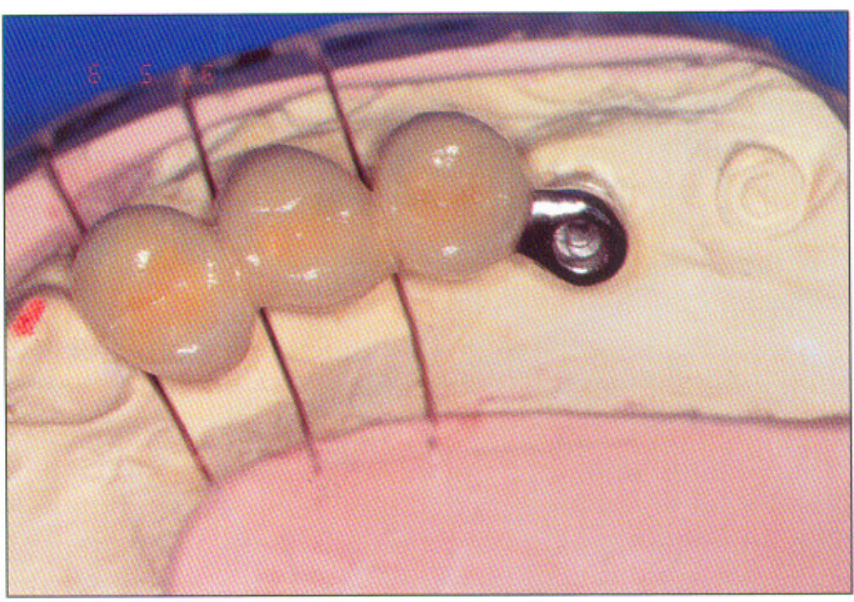

Fig. 6.: Dos implantes, sólo se pudo utilizar el mas anterior ferulizándolo con fundas telescópicas en los premolares.

diente natural de forma que la estructura pudiera ir atornillada al implante y cementada en el diente con un cemento provisional que nos permitiera retirar la prótesis para sus revisiones o ajustes (17). Actualmente, se esta imponiendo la prótesis cementad a en los casos parciales, tanto si el soporte es exclusivamente implantario como si es dentoimplantario, por lo tanto la confección de una cofia telescópica sobre el diente es innecesaria.

Una de las complicaciones mas llamativas de la union diente implante es la intrusión del diente, como han publicado diferentes autores : Ericsson 1986 (18), Cho y Winston 1992 (19), English 1993 (20); Sheets 1993 (21).; Kay 1993 (22) ; Rieder y Parel 1993 (23). Diversas teorias descritas por Rieder 1993 (23), English 1993 (20) y Sheets 1993 (21), tratan de explicar porque se produce esta intrusión, entre las que podemos citar la atrofia por desuso, fenómenos de resonancia, flexion y torsión mandibulares, flexion de la estructura metalica de la prótesis fija, efecto de rebote por la memoria de posición de la estructura metalica, impactacion, etc. Sin embargo, la mayoria de los autores entre los que podemos citar, por haber investigado este fenomeno, a Lindh 1997 (16), Naert 1992 (24) y Olssen 1995 (14) no han encontrado ningun caso con esta complicación.La intrusión del diente natural se produce siempre cuando la conexión entre el diente y el implante es una union no rigida, como señala Lindh (16) si la corona del diente esta cementada de forma permanente, y en particular si la prótesis es soportada por un diente y un implante, el riesgo de intrusión dentaria es minimo.

\section{CONEXIÓN NO RÍGIDA CON ATACHES}

Las restauraciones unidas pasivamente a un diente y a un implante mediante un sistema rompe fuerzas, per- 


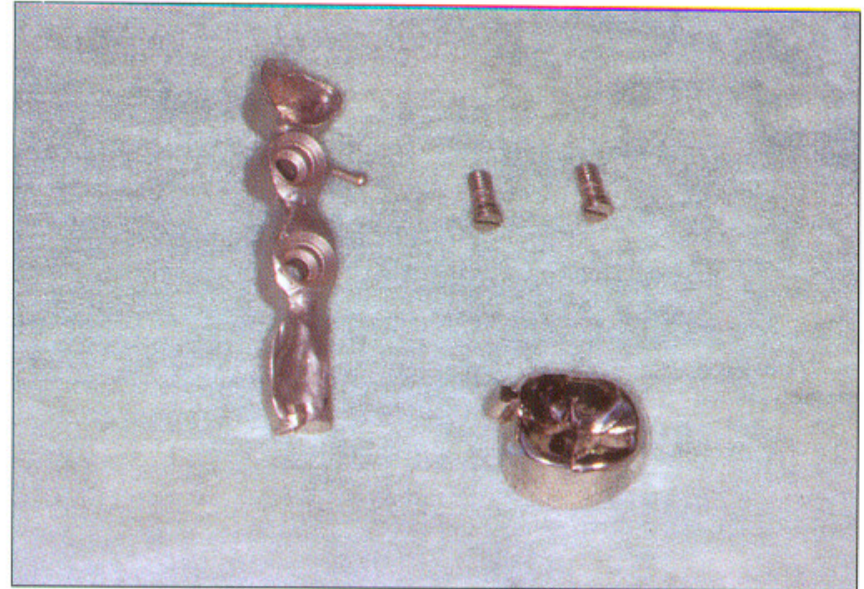

Fig. 1.: Soporte mixto dentoimplantario. Conexión no rígida mediante atache.

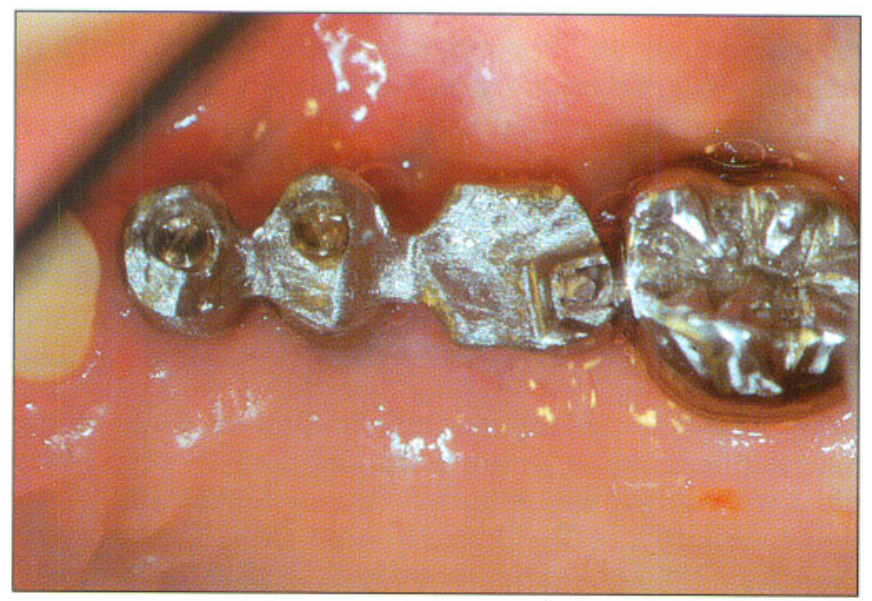

Fig. 8.: Prueba en boca de la estructura metálica.

mite la individualización de las cargas entre ambos pilares, resolviendo, al menos teóricamente el principal problema de estas prótesis, la diferente movilidad del diente y el implante al soportar las cargas oclusales (25) (26). Para algunos autores como Weinberg (27), el conector no rígido permite el movimiento normal del diente y un reparto de las cargas oclusales mas uniforme. También tienen la ventaja de que permiten recuperar parte de la prótesis si uno de los pilares falla.

Skalac (5) y Sullivan (28) aconsejan conexiones no rígidas por los potenciales problemas que las conexiones rígidas pueden ocasionar de sobrecarga del implante, así como una insuficiente carga del diente, que ocasionaría la consiguiente atrofia y extrusión del diente y posiblemente el descementado del pilar natural. Estos problemas se solucionarían mediante la utilización de conexiones no rígidas, ataches con algún grado de libertad de movimientos de forma que permitiera un comportamiento independiente frente a las cargas oclusales.

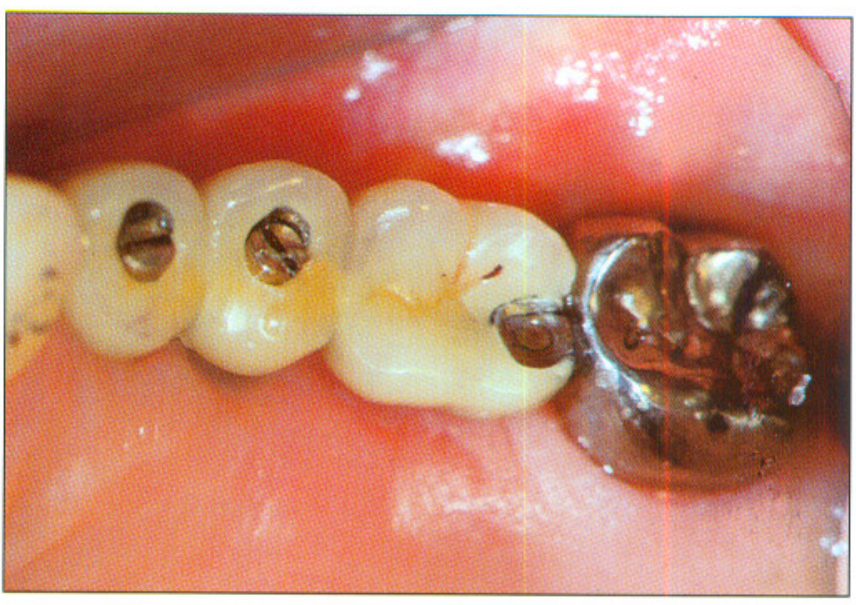

Fig. 9.: Caso terminado. Atornillado a los imp lantes y cementado al diente.

Kay 1993 (22), señala que con estas conexiones no rígidas no siempre se consigue la suficiente ferulizacion del diente, con lo que no se consigue una eficaz distribución de las cargas entre el implante y el diente, incluso la inestabilidad de la conexión puede producir la intrusión del diente, con la consiguiente inestabilidad oclusal. Si esto se produce, todas las cargas oclusales serán soportadas por el implante con el consiguiente riesgo de sobrecarga.

Ademas la experiencia demuestra que los ruptores si no estan perfectamente paralelizados no funcionan correctamente, y que con el uso suelen acumular detritus ocasionando que atache pierda sus movimientos y convierten la conexión en una union rigida.

\section{CONEXIÓN RÍGIDA CON ELEMENTO RESILENTE}

Tratando de encontrar soluciones a la ausencia de ligamento periodontal de los implantes, y tratando de interponer algún elemento que de alguna forma remedara este ligamento y absorbiera parcialmente las cargas que se ejercieran sobre la prótesis, amortiguando las fuerzas que se transmitirían al hueso, es por lo que los implantes IMZ se fabricaban con un elemento intramovil, que es una pieza de politetrafluoruro de etileno con un núcleo de polioximetileno. Es un material viscoelastico que amortigua las cargas oclusales (29).

Con esta pieza intra móvil podemos compensar la disparidad de intrusión del diente y del implante, por lo tanto este implante seria favorable para ser utilizado en combinación con un diente natural, ya que conseguiríamos un comportamiento biomecánico similar entre ambos pilares. Estos implantes permitirían ser ferulizados rígidamente a un diente.(30) 


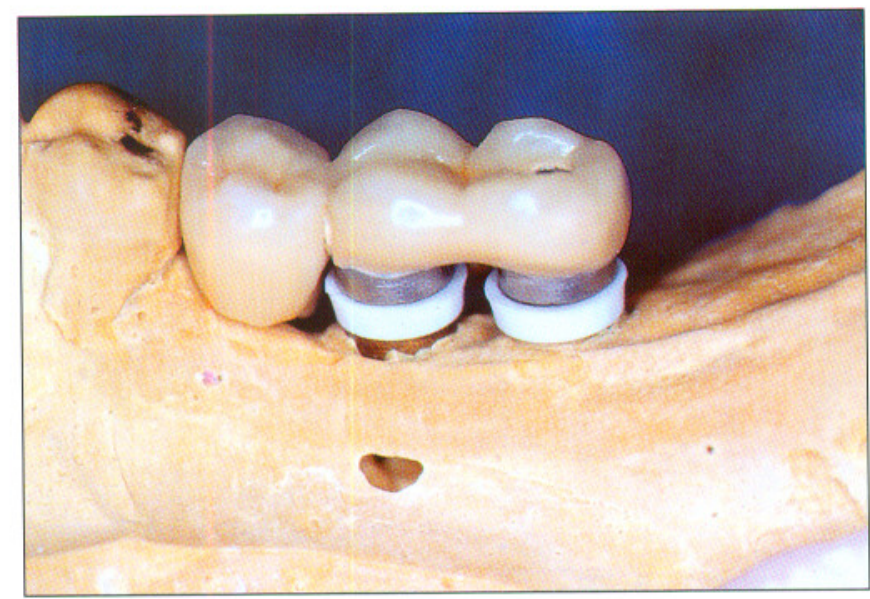

Fig. 10.: Prótesis con elemento resilente.

Revisando la literatura se encuentran resultados muy diferentes respecto a la eficacia de estos implantes con pieza intramovil. Babush (31)(en una revisión de 3436 implantes IMZ, de los que el 34\% llevaban mas de 5 años colocados, presento un porcentaje de éxito del $97,5 \%$ y solamente encontro fracturas del elemento intramovil en el $1,4 \%$.

Otros autores como Hobo (32), hace referencia en su libro, a las frecuentes fracturas del elemento intramovil,que hacen necesaria su muy frecuente sustitución, aunque no cita ningun estudio estadístico.

Ackerman, en un seguimiento a 12 años de 5000 implantes IMZ, presenta un éxito del $97 \%$, un $75 \%$ de ellos en casos parciales y muchos de ellos ferulizados a dientes naturales.

Kay (21)sugiere la conveniencia de utilizar implantes con elemento intramovil para ferulizarlos rígidamente al diente natural.

Como en otras ocasiones, a pesar de que las publicaciones científicas respaldan la conveniencia de utilizar implantes con elemento intramovil y de publicarse las escasas complicaciones, pequeño porcentaje de complicaciones, sin embargo, la experiencia mas comun parece indicar que el porcentaje de problemas con el elemento intramovil u otros mecanismos que aporten resilencia al implante es mucho mayor que si no lo tienen y esto ha condicionado la utilización de los implantes con pieza intramovil.

\section{CONSECUENCIAS DE UNIR RÍGIDAMENTE DIENTE E IMPLANTE}

Los estudios in vivo publicados por Jemt en 1989 (33)

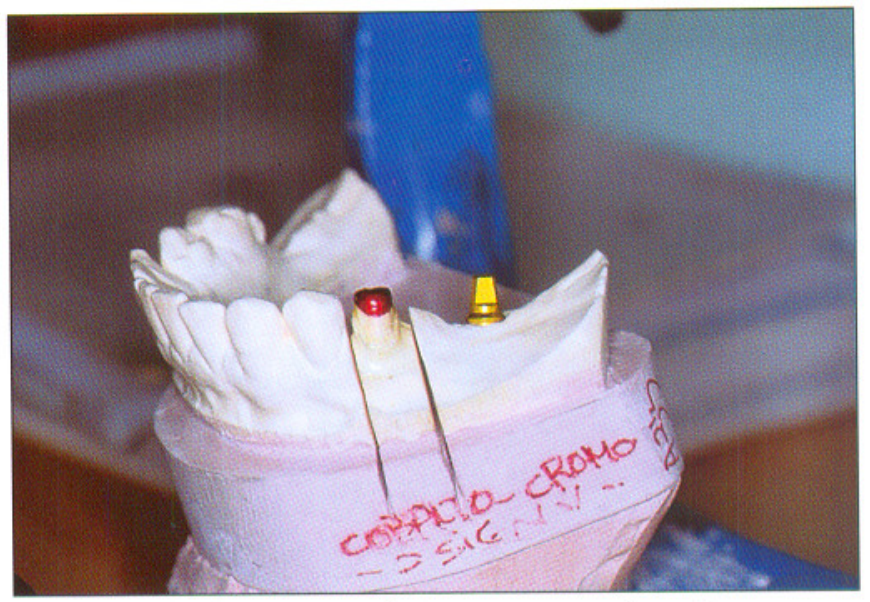

Fig. 11.: Un implante con un diente para reponer tres piezas.

en una investigación hecha sobre 876 implantes colocados consecutivamente en pacientes desdentados parciales no encontro diferencias significativas. Olssen et al , 1995 (14) y Richter en 1995 (34) en estudios realizados in vivo demostraron que no se producían sobrecargas en los implantes cuando los implantes se ferulizaban rígidamente a los dientes, en una prótesis de tres piezas. Un estudio publicado por van Steenberghe (35) evaluando 133 implantes colocados en 38 pacientes parcialmente edéntulos no encontró problemas ni mecánicos ni periodontales, con un seguimiento de los casos entre 6 y 36 meses. Gunne et al. Publicaron en 1997 (36) un estudio en el que trataron a pacientes desdentados parciales clase I de Kennedy mandibular, en un lado se colocaban dos implantes con una prótesis fija implantosoportada y en el otro lado se colocaba un solo implante realizando una prótesis fija dentoimplantosoportada. Obtuvieron como conclusión que no había diferencia entre el pronóstico de uno y otro lado, que no se producía diferencia en la distribución de cargas entre uno y otro lado. La distribución de las cargas entre los dos pilares de soporte sean los dos implantes o implante y diente dependía mas de la geometría de la prótesis y a la disposición del pilar que al hecho de ser diente o implante, concluye que la razón de esta distribución compartida de las cargas se debe a que la intrusión del diente es parcialmente compensada por la flexibilidad del implante unitario. Destacan los autores que esta posibilidad de palanca no se producirá cuando tengamos múltiples implantes ferulizados entre si. Rangert et al.(37) , en un análisis in vitro concluian que se podía unir un implante Branemark con un diente debido a la flexibilidad del implante que compensaría la movilidad del diente. Clepper 1997 (38), en un estudio retrospectivo de 103 implantes Paragon colocados consecutivamente en mandíbulas parcialmente edéntulas combinando dos dientes con implante mediante prótesis fija cementada, obteniendo buenos resultados y conclu- 


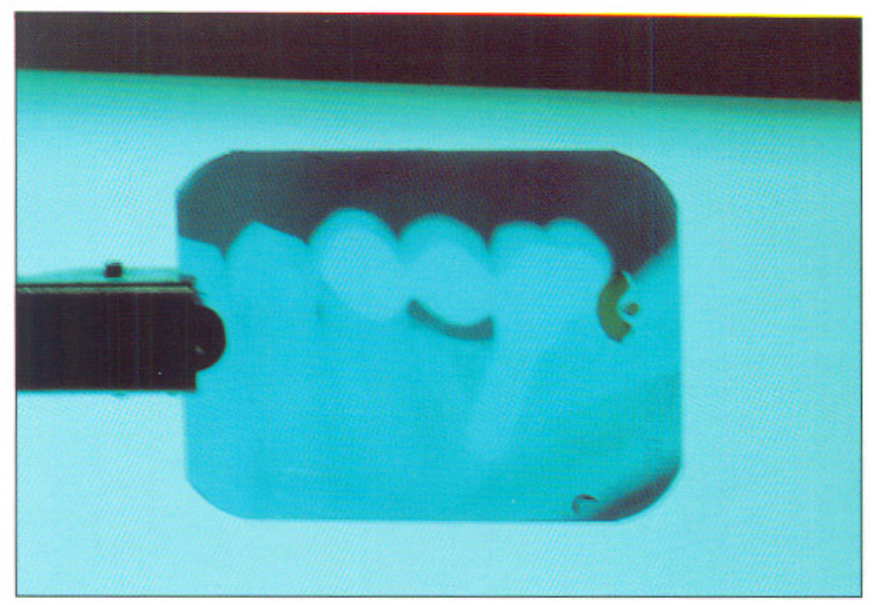

Fig. 12.: Unión rigida atornillada al implante y cementada al diente.

ye que deberíamos evitar como soporte aquellos dientes que presenten una mala proporción corona /raiz, o aquellos dientes con grandes espigas muñón., la conexión diente implante es normalmente el modo más práctico de tratar a nuestros pacientes. Gunne publicó en 1999 (39) un informe de 10 años de experiencia con prótesis fijas de soporte mixto, diente e implante, deduce que una prótesis fija soportada por un diente y un implante es un tratamiento alternativo, predecible y fiable en la zona mandibular posterior.

Los estudios clínicos longitudinales demuestran que no existe ninguna consecuencia negativa de unir un diente a un implante con excepción de algún caso de intrusión radicular, descrito en un porcentaje muy pequeño de tratamientos, en todos los casos la unión diente implante se hizo con conectores no rígidos. Ericsson et al., 1986 (18); Cho G.C. y Winston WLC 1992 (19), Kirsch y Mentag, 1986 (8); Brunski 1998 (4); Jemt et al. , 1989 (33); Patrick et al. , 1989 (40); Kent et al. , 1990 (41); Naert et al. , 1992 (24); Reider y Parel , 1993 (23). English C.E. 1993 (20). Sheets C.G. y Earthman JC 1993 (21).Sin embargo, la mayoria de los autores no han tenido ningun efecto adverso en los pilares, tanto en el implante como en el diente natural, incluyendo la intrusión dentaria. Si la corona del diente natural esta cementada de forma definitiva, y si la prótesis esta unicamente soportada por un implante y un diente, el riesgo de intrusión es minimo.

Los estudios experimentales siempre han sido menos favorables a admitir esta unión rígida entre elementos tan diferentes, pero las ultimas publicaciones de análisis hechos en el laboratorio, obtienen conclusiones no tan radicalmente diferentes o concluyentes, sobre esta posibilidad. Nishimura en 1999 (42), publico que la conexión rígida entre un diente y un implante solamente ocasiona una ligeramente mayor tensión en el implante que si la conexión es no rígida, y que ambas conexiones mostraban en este estudio de análisis de

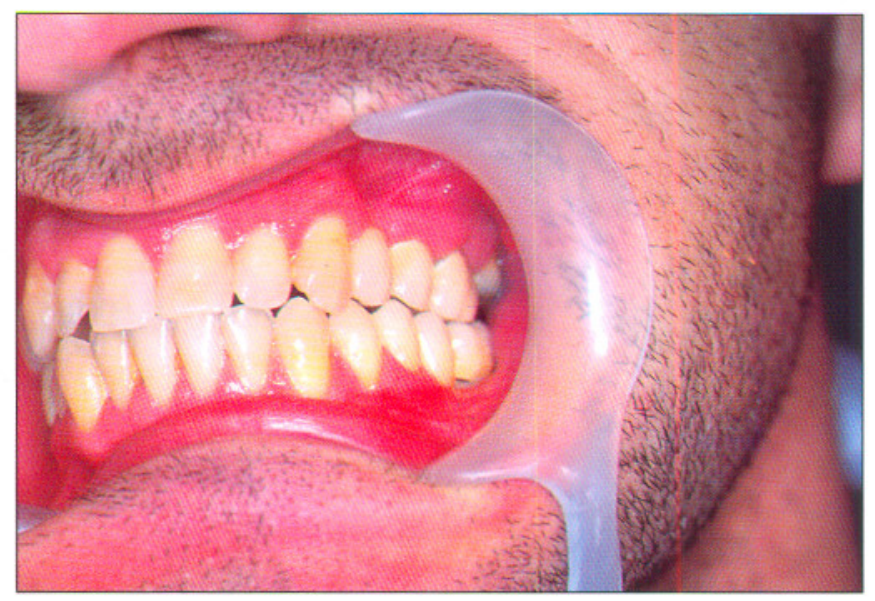

Fig. 13.: Unión rígida cementada al diente y al implante.

distribución de cargas en un modelo de fotoelasticidad, una adecuada distribución de cargas entre los pilares de soporte.

El dilema de unir implante y diente se puede obviar colocando suficiente número de implantes, que nos permitan realizar una prótesis de soporte exclusivamente implantario (43). Sin embargo, hay muchas situaciones clínicas en las restauraciones de los parcialmente desdentados en las que la unión de un diente con un implante sería conveniente. En este artículo nos estamos refiriendo al tratamiento de extremos libres mandibulares, esto no quiere decir que no existan otras indicaciones para poder unir dientes e implantes, pero debemos ser muy cuidadosos en no generalizar los resultados obtenidos en determinadas zonas con los posibles resultados en otras partes de los maxilares. Las situaciones clínicas que nos llevan a unir diente con implante en extremo libre madibular, son aquellos casos en que no podemos colocar mas implantes, sobre todo si atendemos a las indicaciones de algunos autores de colocar tres implantes no alineados (44); en algunas ocasiones sólo podemos colocar un implante situado por delante del agujero mentoniano, porque debido a la extrema reabsorción del proceso alveolar podríamos dañar el nervio dentario, como describe Lindh. Se ha establecido que una arcada acortada, con los incisivos y las caras oclusales de los premolares, se mantiene una suficiente función oral .( Witter 1994) (45),(46). En otras ocasiones aunque se pueda colocar un implante por encima del nervio dentario, puede ser arriesgado colocar un segundo implante. En otras ocasiones podemos encontrarnos que aunque se hubieran colocado dos implantes uno de ellos ha fracasado, y disponemos sólo de otro. Una consideración menos académica pero sin embargo de trascendencia clínica es el factor económico, dado que el tratamiento con un solo implante unido al diente se abarata sustancialmente los costes 


\section{CONCLUSIONES}

A pesar de que los estudios de análisis mecánicos realizados in vitro demuestran las desfavorables consecuencias de la ferulizacion rígida entre diente e implante, la información clinica disponible hasta el momento demuestran que los porcentajes de éxito y de supervivencia de estos implantes unidos a dientes no presentan mas complicaciones que si estuvieran unidos a otro implante, aparte de los pocos casos descritos de intrusión dentaria.

Los análisis mecánicos sobre el comportamiento y las ventajas de utilizar elementos que compensen la diferencia de resilencia entre el diente y el implante, demuestran su efectividad reduciendo las cargas recibidas por el hueso periimplantario. Sin embargo, los resultados clinicos no demuestran la eficacia de estos elementos de absorción de cargas, en reducir la concentración de tensiones en el hueso que rodea al implante. Dado que no resultan muy utiles los procedimientos que tratan de otorgar una cierta capacidad de movilidad, de absorción de cargas al implante, la otra alternativa para tratar de compensar la diferencia de resilencia es disminuir la movilidad del diente natural. Disponemos de diferentes procedimientos para conseguir este objetivo, como es endodonciar el diente persiguiendo el efecto secundario de su anquilosis al hueso y por lo tanto disminuyendo drásticamente su movilidad, otra posibilidad es ferulizar varios dientes entre si mediante una prótesis rígida que tambien se uniria al implante. Las ventajas de estas alternativas requieren confirmación con posteriores estudios de análisis mecánicos y de evaluación clinica, tendremos que sopesar los posibles beneficios de estas técnicas con las posibles consecuencias iatrogénicas y el mayor coste económico que pueden conllevar la preparación de multiples dientes para cubrir con coronas de recubrimiento total.

Indudablemente la conexión diente implante es un tema muy debatido, con conceptos biomecánicos y clinicos en continuo cambio, que implican una permanente puesta al dia. Hasta que las investigaciones de laboratorio y sobre todo los ensayos clinicos a largo plazo sean definitivos con respecto a este tema y los clinicos tengamos que seguir enfrentándonos con la resolución de los problemas de nuestros pacientes de la mejor forma posible, tenemos que ser concientes que hoy por hoy las decisiones deberemos tomarlas de una forma un tanto intuitiva y empírica.

Teniendo en cuenta lo anteriormente expuesto, nos atrevemos a presentar la pauta que actualmente seguimos en el servicio de implantoprotesis de la Facultad de Odontología de la Universidad Complutense de Madrid:
Valorar las circunstacias concretas de cada paciente, prefiriendo el soporte exclusivamente implantario siempre que sea posible, aunque cada vez se tiene mas en consideración la posibilidad de restaurar un extremo libre inferior, a partir del primer premolar, mediante la colocación de un solo implante.

El implante se colocara aproximadamente a $15 \mathrm{~mm}$. del diente (prótesis de tres piezas)

Ferulizaremos mediante una prótesis rígida diente e implante.

Utilizaremos de soporte,solo un diente y un implante

El diente no es necesario que sea endodonciado.

\section{SUMIMARY}

The problem founded when restoring partially edentulous patients with osseointegrated implants is whether the implants should be connected to, or separated from the natural teeth. This dilemma is caused by the difference in mobility of teeth and osseointegrated implants. Brunski reported differences in intrusive stiffness for implants and teeth.

However, there are several benefits for implants used in conjunction with natural teeth, which include: reduction of the number of implants needed for restoration, lower cost and maintenance of proprioception.

This article presents a review on biomechanical aspects and some considerations of splinting teeth and implants, rigid and no rigid connections between implants and teeth are discussed. There are several areas of interest regarding the connection of implants and natural teeth, wich may provide different amounts of support depending on periodontal status. For a particular clinical situation, the selection of the tipe of the restorative interproximal connection, and quality of implant and teeth support are main issues.

\section{KEY WORDS}

Dental implants, Fixed partial prostheses, combination with natural teeth. 


\section{BIBLIOGRAFÍA}

1. BRANEMARK PL., ZARB G. ALBREKTSSON T. Protesis tejido-integradas. Ed. Quintessence Verlags-GmbH 1987.

2. COHEN SR, ORENSTEIN JH. The use of attachments in combination implant and natural tooth fixed partial dentures. A technical report. Int. J. Oral Maxillofac. Implants 1994; 9: 230-234.

3. SCHNITMAN PA. Implants for partial edentulism. J. Dent. Educ. 1988; 52(12): 725-736.

4. BRUNSKI J. Biomechanics of oral implants future research directions. J Dent Ed 1988; 52: 775-87.

5. SKALAK R. Aspects of biomechanical considerations. In Branemark P-I, Zarb GA, Albrektsson T (eds), TissueIntegrated Prostheses. Chicago. Quintessence, 1985: $117-128$

6. UHLRICH, R., MUHLBRANDT, L., MOHLMANN, H. \& SCHMID, M. (1993) Qualitative mechano-perception of natural teeth and endosseous implants. International Journal of Oral and Maxillofacial Implant, 8, 173.

7. HAMMERLE, C. H. F., WAGNER, D., BRAGGER, U., LUSSI, A., KARAYIANNIS, A., JOSS, A. \& LANG, N.P. (1995) Therhold of tactile sensitivity perceived with dental endosseous implants and natural teeth. Clinical Oral Implants Research, 6, 83.

8. KIRSCH, A. \& ACKERMANN, K.L. (1989) The IMZ osseintegrated implant system. Dental Clinics of North America, 33, 733.

9. RICHTER, E-J. (1989) Basic biomechanics of dental implants in prosthetic dentistry. Journal of Prosthetic Dentistry, 61, 602 .

10. RICHTER, E-J., ORSAY, B. \& JOVANOVIC, S.A. (1990) Dental implant abutment resembing the two-phase tooth mobility. Journal of Biomechanics, 23, 297.

11. VALENTIN, I., TAIEB, A., BENHAIM, L., FRANQUIN, J-C \& HOURI, A. (1990) Analyse de la repartition des contraintes osimplant. Cahier Prostheses, 72, 143.

12. VAN ROSSEN, I.P., BRAAK, L.H., DE PUTTER, C. \& DE GROOT, K. (1990) Stress-absorbing elements in dental implants. Journal of Prosthetic Dentistry, 64, 198.

13. KIRSCH, A. \& MENTAG, P. (1986) The IMZ endosseous two phase implant systems: a complete oral rehabilitation treatment concept. Journal of Oral Implantology, $12,576$.

14. OLSSEN, M., GUNNE, J., ASTRAND, P. \& BORG, K. (1995) Bridges supported by free standing implants vs. Bridges supported by tooth and implants. $A$ five year prospective study. Clinical Oral Implants Research, 6, 114.

15. ALTAY, O.T., TSOLKA, P. \& PRIESKEL, H. (1990) Abutment teeth with extra coronal attachments: The effects of splinting on tooth movement. International Journal of Prosthodontics, 3, 441 .

16. LINDH T, GUNNE J, DANIELSSON S. Rigid conections between natural teeth and implants: a tecnical note. Int. J. Oral Maxillofac. Implants 1997; 12: 674-678.

17. RICHTER, E-J, SPIEKERMAN, H. \& JOVANOVIC S.A. (1990) Tooth to implant fixed prótesis: Biomechanics based on in vitro and in vivo measurements. In: Tissue Integration (eds. W.R. Laney \& D. E. Tolman), p. 133. Quintessence Publishing Co., Chicago.

18. ERICSSON, I., LEKHOLM, U., BRANEMARK, P-I., LINDHE, J., GLANTZ, P-O \& NYMAN, S. (1986) A clinical evaluation of fixed-bridge restorations supported by combination of teeth and osseo-integrated titanium implants. Journal of Clinical Periodontology, 13, 307.

19. CHO GC, WINSTON WLC. Apparent intrusión of natural teeth under an implant-supportes prosthesis: A clinical report. J Prosthet Dent 1992; 68: 3-5.

20. ENGLISH, C.E. (1993) Biomechanical concerns with fixed partial dentures involving implants. Implant Dentistry, 2, 22.

21. SHEETS CG, EARTHMAN JC. Natural tooth intrusion and reversal in implant-assisted prótesis: Evidence of and a hipótesis for the occurrence. J Prosthet Dent 1993; 70: $13-20$.

22. KAY HB. Ossointegration-beyond tooth replacement; the intramobile cylinder (IMZ) the intramobile cylinder as a stabilinzing abutment in periodontal-prosthesis. Int J Periodont Rest Dent 1993; 13:394-415.

23. RIEDER, C.E. \& PAREL, S. M. (1993) A survey of natural tooth abutment intrusion with implant-connected fixed partial dentures. International Journal of Periodontology and Restorative Dentistry, 13, 335.

24. NAERT, I., QUIRYNEN, M., VAN STEENBERGHE, D. \& DARIUS, P. (1992) A six year prosthodontic study of 509 consecutively inserted implants for the treatment of partial edentulism. Journal of Prosthetic Dentistry, 67, 236.

25. RODRÍGUEZ MARTINEZ A, RODRÍGUEZ MARTINEZ F, GAGOE. Rehabilitación mediante una prótesis dentoimplanto-soportada con un interlock en su union. Rev.Esp Odontoestomatologica de implantes 1997; 5-4, 181-4.

26. KAYAKAN R, AALLARINI R, MULLEN R. Theoretical study of the effects of tooth and implant mobility differences occlusal force transmisión in tooth/implant-supported partial prostheses. J Prosthet Denta 1997; 78: 391-99.

27. WEINBERG LA. The biomechanics of force distribution in implant-supported bridges. International Journal of Oral and Maxillofacial Implants 8: 1931.

28. SULLIVAN DY. Prosthetic considerations for the utilization of osseointegrated fixures in the partially edentulous arch. Int J Oral Maxillofac Implants 1986; 1: 39-45. 
29. McGLUMPHY EA, CAMPAGNI WW, PETERSON LJ. A compaison of the stress transfer characteristics of a dental implant with a rigid or a resilient internal element. J Prosthet Dent 1989; 62: 586-93.

30. HOSHAW, S.J. \& BRUNSKI, J.B. (1988) Mechanical testing of dental implants with and without "Intramobile Elements". Journal of Dental Research, 67 Special Issue, 314 (Abstract No. 1612).

31. BABBUSH D, KIRSCH A, MENTAG PJ, HILL B, Intramobile cylinder (IMZ) two stage osteointegrated implant system with the intramobile element (IME). Part I: Its rationale and procedure for use. Int J Oral Maxillofac Implants 1987; 2: 203-216.

32. HOBO S, ICHIDA E, GARCIA LT. Osteointegracion y rehabilitación oclusal. Ed. Marb libros. Madrid 1997.

33. JEMT, T., LEKHOLM, U. \& ADELL, R. (1989) Osseointegrated implants in the treatment of partially edentulous patients: a preliminary study on 876 consecutive placed implants. International Journal of Oral Maxillofacial Implants, 4, 211.

34. RICHTER, E-J. (1995) In vivo vertical forces on implants, International Journal of Oral and Maxillofacial Implants, 10,99 .

35. VAN STEENBERGHE, D. (1989). A retrospective multicenter evaluation of the survival rate of osseointegrated fixtures supporting fixed partial prostheses in the treatment of partial edentulism. Journal of Prosthetic Dentistry, 61, 217.

36. GUNNE J, RANGERT B, GLANTZ PO, SVENSSON A. Functional loads on freestanding and connected implants in three-unit mandibular prostheses opposing complete dentures: An in vivo study. Int J Oral Maxillofac Implants 1997; 12: 335-341.

37. RANGERT B, GUNNE J, GLANTZ P-O, SVENSSON A. Vertical load distribution on a three-unit prosthesis supported by a natural tooth and a single Branemark implant. Clin Oral Implants Res 1995; 6: 40-46.
38. CLEPPER DP. Should natural teeth and osseointegrated implants be used in combination to support a fixed prosthesis? The International Journal of Oral \& Maxillofacial Implants.

39. GUNNE J, ASTRAND P, LINDH T, BORG K, OLSSEN M. Dentaduras parciales fijas soportadas por dientesimplantes y por implantes: informe de 10 anos . Int. J. Prosthodont 1999; 12: 216-221.

40. PATRICK D, ZOSKY J, LUBAR R, BUCHS A. The longitudinal clinical efficacy of Core-Vent dental implants: a five years repor. Journal of Oral Implantology. 1989; 15,95.

41. KENT, J.N., BLOCK, M.S., FINGER, I.M., GUERRA, L., LARSEN, H. \& MISIEK, D.J. (1990). Biointegrated hydroxylapatite-coated dental implants: 5-year clinical observations. Journal of the American Dental Association, 121, 138.

42. NISHIMURA R, OCHIAI K.T., CAPUTO A, JEONG C.M. Photoelastic stress analysis of load transfer to implants and natural teeth comparing rigid and semirigid connectors. J. Prosthet Dent 1999; 81: 696-703.

43. DEL RIO HIGSMITH J, SADA GARCIA-LOMAS J. Consideraciones prostodoncicas y biomecánicas en prótesis parcial fija sobre implantes endooseos. Rev. Europea de Odontoestomatologia. 2, Abril 90, 89-96.

44. BRUNSKI J. Biomechanics of dental implants. In: Block M, Kent J, editors. Endosseous implants for maxillofacial reconstruction. Philadelphia:WB Saunders; 1995.

45. WITTER DJ, DE HAAN AFJ, KAYSER AF, VAN ROSSUM GMJM. A 6-year follow-up study of oral function in shortende dental arches. Part I: Occlusal stability. J Oral Rehabil 1994; 21: 113-125.

46. WITTER DJ, DE HAAN AFJ, KAYSER AF, VAN ROSSUM GMJM. A 6-year follow-up study of oral function in shortened dental arches. Part II: Craniomandibular dysfunction and oral comfort. J Oral Rehabil 1994; 21:353-366. 